ever, other crystallographic surface planes are also observed. Atomic-level imaging and spectroscopy of those planes allowed the researchers to identify a surface $\mathrm{CuO}_{2}$ plane.

"In contrast to previous studies, we found that this copper oxide layer exhibits an unusual suppression of tunneling conductance at low energies," Yazdani said. "We think the orbital symmetry of the plane's electronic states may be influencing the tunneling process and is responsible for the strange behavior we observed at the surface."

Surface-sensitive techniques, such as electron tunneling and photoemission, have been crucial in gleaning information about high-temperature superconductors, Yazdani said. But it has not always been clear from which layer the information was coming. By imaging at the atomic scale and probing on the nanoscale, the researchers achieved much higher precision.

"High-temperature superconductors are layered compounds containing one or more copper oxide planes and other layers that act as charge reservoirs," Yazdani said. "Like dopants in a semiconductor, these layers donate charge carriers to the copper oxide planes, making them conducting. The strong electronic interactions in the copper oxide planes are responsible for the material's unusual electronic properties."

By exploring large areas of the sample and correlating the STM topographic images with $x$-ray crystallographic data, the researchers were able to identify individual layers of copper oxide and of bismuth oxide and then measure their electronic properties.

"With the STM, we can send electrons through the tip and measure the rate at which they flow into the surface," Yazdani said. "We found a very strong contrast in the spectra taken on the two surfaces. The electron tunneling in the copper oxide plane was strongly suppressed at low energies."

This behavior is unexpected in a $d$ wave superconductor, Yazdani said, and could demonstrate the dramatic influence of the layered structure on the surface electronic properties. The observations can best be explained by the way in which the STM tip couples to the electronic states of the copper oxide plane, the researchers concluded.

"At low energies, electrons from the tip are constrained by the orbital symmetry of the plane's electronic-wave function, which resembles a cloverleaf pattern," Yazdani said. "This directional dependence of the current can explain the suppressed tunneling."

\section{Neutron-Scattering Technique Developed to Characterize Nanoporous Thin Films}

Researchers from the National Institute of Standards and Technology (NIST) reported on August 20 at the American Chemical Society meeting in Boston that they have developed methods for characterizing key structural features of porous films under consideration as insulators for the ultrathin metal wires in integrated circuits. The advance will help semiconductor manufacturers and their materials suppliers identify the most promising "nanoporous film" candidates for shield- ing miles of interconnecting wire on nextgeneration microprocessors.

To increase processor speed, semiconductor manufacturers seek to reduce chip sizes. However, size reduction introduces problems with electrical interference between circuit elements (crosstalk), motivating the development of better insulating materials. Current insulating materials such as silicon dioxide and fluorinated silicate glass (FSG) are approaching their limits as devices are squeezed closer together on a chip.

To prepare better insulating films, many materials suppliers are developing films

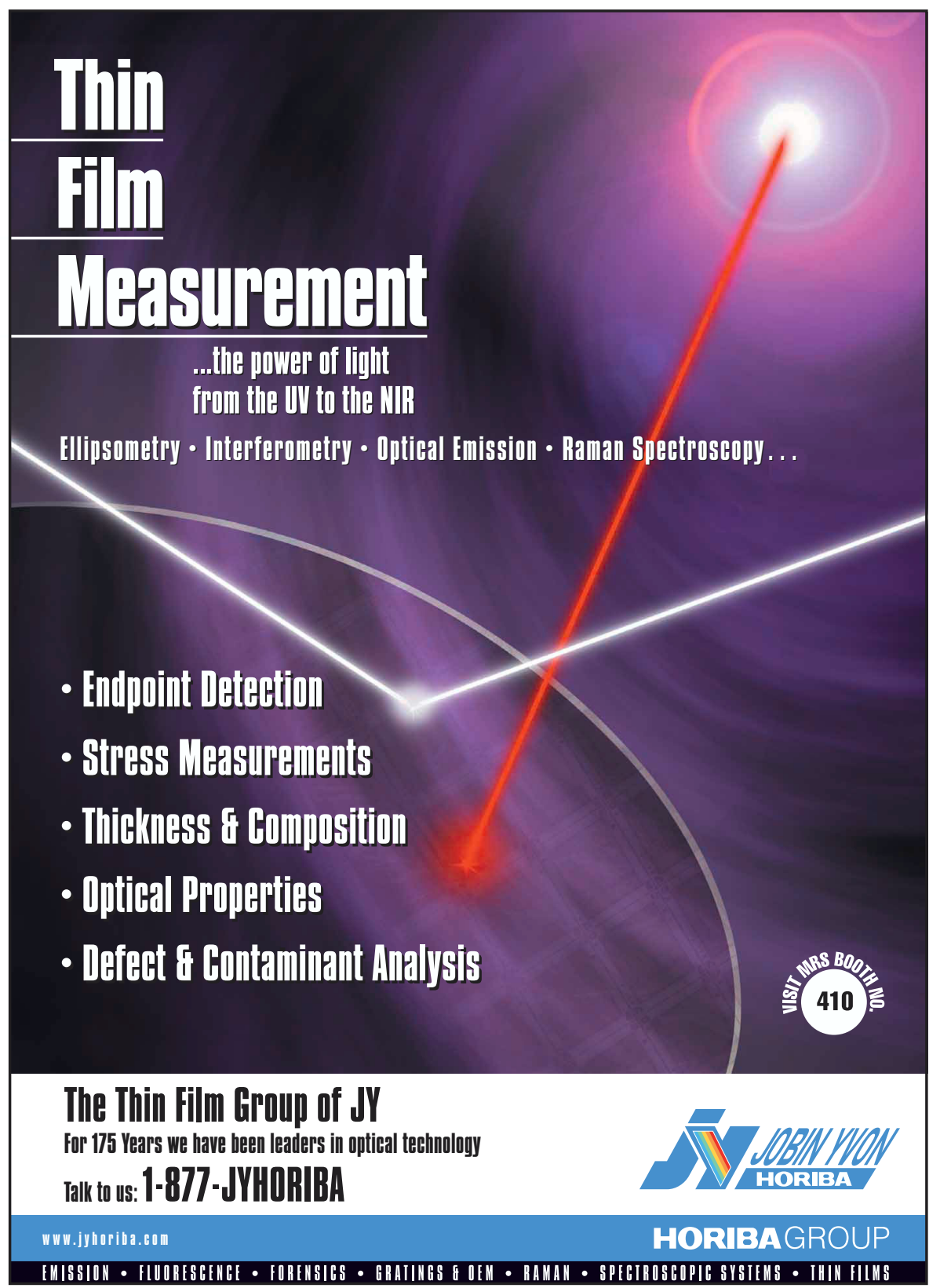

Circle No. 19 on Inside Back Cover 
interspersed with very small holes that measure $\leq 5 \mathrm{~nm}$ in diameter. Introducing nanometer-sized air bubbles lowers a material's dielectric constant, $\kappa$. Air, the ideal insulator, has a $\kappa$ value of 1 . Silicon dioxide and FSG, in contrast, have $\kappa$ values of about 4.2 and 3.7 , respectively.

For several years, the NIST team has been performing a variety of measurements to help the semiconductor industry characterize potential nanoporous insulators. Working with the NIST Center for Neutron Research, materials scientists Ronald Hedden, Barry Bauer, and Hae-Jeong Lee of the NIST Polymers Division developed a neutron-scattering technique for surveying minuscule holes in film samples supplied by International SEMATECH, a consortium of chip makers. Combined with information gathered with other methods, neutron-scattering measurements reveal the size and volume fraction of pores, the connectivity among pores, and the density of the underlying matrix.
This approach to developing new insulators presents some formidable challenges. Riddling a material with tiny holes (or bubbles) lowers its $\kappa$ value, but changes other important properties as well. Also to be taken into account, for example, are a candidate material's strength and hardness, how well it adheres to different substrates, and whether it can withstand high temperatures as well as etch chemicals during processing.

Bauer said, "The more fully we can characterize the pore structure and properties of these nanoporous materials, the more straightforward the search becomes."

Bauer, Hedden, and Lee led an effort to extend contrast-matching-a neutronbased technique for studying bulk materials-to nanoporous thin films. They succeeded by pumping solvent vapor mixtures into a special flow-through chamber containing the films. The vapor condenses into the pores, permitting neutron-scattering measurements that probe the film density while yielding valuable informa- tion about pore connectivity. The new neutron technique also can detect nanometersized inhomogeneities in the composition of the matrix.

Though the results are promising, the contrast-matching procedure is timeconsuming, taking three to four days per sample. One goal of the team is to develop a simpler alternative that industry could use to characterize films faster and more cheaply. Hedden said neutronscattering measurements could be used as a benchmark to evaluate data gathered with other approaches.

\section{FOR MORE RESEARCI NEWS ON MATERIALS SCIENCE . . . \\ ... access the Materials Research Society Web site:}

www.mrs.org/gateway/ matl_news.html

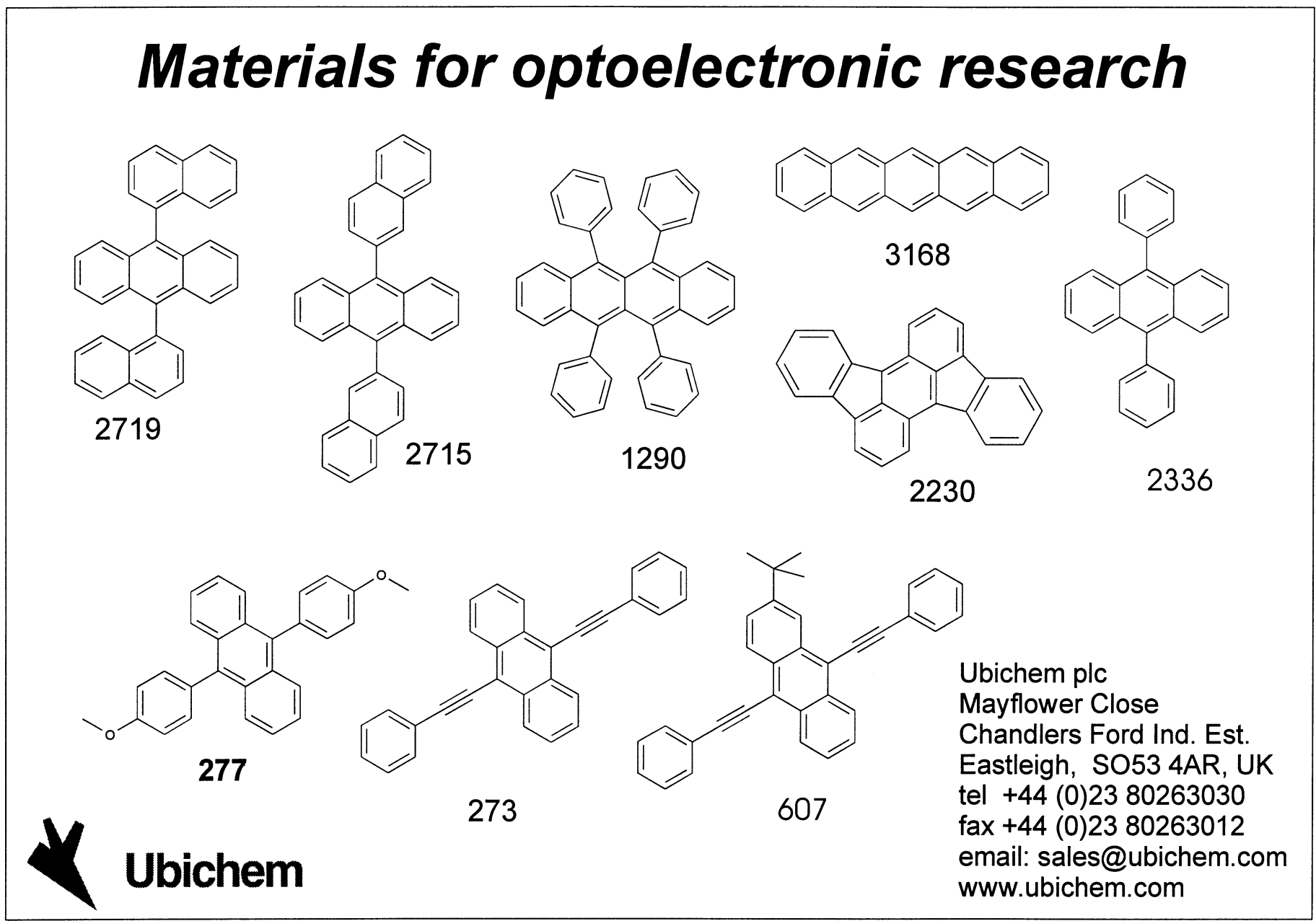

Circle No. 34 on Inside Back Cover 